\title{
Hydrodynamic instabilities in astrophysics and in laboratory high-energy-density systems
}

\author{
R P Drake \\ Department of Atmospheric, Oceanic and Space Science, University of Michigan, Ann Arbor, \\ MI 48109, USA \\ E-mail: rpdrake@umich.edu
}

Received 1 July 2005

Published 8 November 2005

Online at stacks.iop.org/PPCF/47/B419

\begin{abstract}
High-energy-density systems and astrophysical systems both involve hydrodynamic effects, including sources of pressure, shock waves, rarefactions and plasma flows. In the evolution of such systems, hydrodynamic instabilities naturally evolve. As a result, a fundamental understanding of hydrodynamic instabilities is necessary to understand their behaviour. This paper discusses the validity of a hydrodynamic description in both cases, and, from the common perspective of the basic mechanisms at work, discusses the instabilities that appear in astrophysics and at high energy density. The high-energy-density research facilities of today, built to pursue inertial fusion, can accelerate small but macroscopic amounts of material to velocities above $100 \mathrm{~km} \mathrm{~s}^{-1}$, can heat such material to temperatures above $100 \mathrm{eV}$ and can produce pressures far above a million atmospheres $\left(10^{12} \mathrm{dyn}^{-2}\right.$ or $\left.0.1 \mathrm{TPa}\right)$. In addition to enabling inertial fusion research, this enables these facilities to do experiments under the conditions that address basic physics issues including issues from astrophysics. One can devise experiments aimed directly at important processes such as the Rayleigh Taylor instability at an ablating surface or at an embedded interface that is accelerating, the Richtmeyer Meshkov evolution of shocked interfaces and the Kelvin-Helmholtz instability of shear flows. The paper includes examples of such phenomena from the laboratory and from astrophysics.
\end{abstract}

(Some figures in this article are in colour only in the electronic version)

\section{Introduction}

If one browses in the literature of astrophysics and inertial fusion, one finds remarkably similar terms and concepts describing the evolution of these systems. In particular, one finds hydrodynamic phenomena such as shock waves, rarefactions and instabilities, including the Rayleigh-Taylor instability [1,2]. The astrophysical applications of hydrodynamic concepts 
extend far beyond the interior of stars, to supernova remnants (see, e.g. Chevalier et al [3] or Jun et al [4]) and to other systems evolving in magnetized plasmas that are manifestly collisionless. It may not be obvious whether such applications are truly valid or whether they just represent the least bad analysis that can be done. The inertial fusion applications largely involved ionized matter, so that it is unclear $a b$ initio to what degree plasma effects may alter the hydrodynamic behaviour.

If one looks a little further one can find that Takabe in 1993 [5] suggested the use of inertialfusion facilities to learn more about the hydrodynamic instability processes in supernovae, and that during the next decade a wide range of related activities developed as reviewed in [6-9].

The present paper is devoted to seeking common ground among these areas of research. In the following, we first consider whether and when a hydrodynamic description is valid in such systems. Once we establish that such a description can be worthwhile, we proceed to consider the hydrodynamic instabilities and the related processes that such systems exhibit. These break naturally into three categories, reflecting three origins of the development of structure. The first category is buoyancy, which is responsible for the ubiquitous Rayleigh-Taylor instability. The second category is lift, which is responsible for the Kelvin-Helmoltz instability and related mechanisms involved, for example, in the destruction of clumps. The third category we might label 'vorticity generation and transport', as this can create substantial structure even in the absence of unstable dynamics in the usual sense and can also greatly complicate the structures produced by the two fundamental driving forces just mentioned.

\section{Why hydrodynamics and is this valid?}

Hydrodynamics, in its simplest form, is the behaviour of systems that can be accurately described by the Euler equations, specifically

$$
\begin{aligned}
& \frac{\partial \rho}{\partial t}+\nabla \cdot \rho \boldsymbol{u}=0, \\
& \rho \frac{\partial \boldsymbol{u}}{\partial t}+\boldsymbol{u} \cdot \nabla \boldsymbol{u}=-\nabla p
\end{aligned}
$$

and

$$
\frac{\partial p}{\partial t}+\boldsymbol{u} \cdot \nabla p=-\gamma p \nabla \cdot \boldsymbol{u}
$$

where $\boldsymbol{u}, \rho$ and $p$ are the velocity, density and pressure, respectively. Equation (3) assumes that the fluid is polytropic (e.g. see [10]), so $p \propto \rho^{\gamma}$, where $\gamma$ is the adiabatic index (the ratio of specific heats). For a fully ionized non-relativistic gas (at high enough temperature) $\gamma$ is equal to 5/3; for a gas where radiation pressure is dominant, $\gamma$ is equal to $4 / 3$; for a diatomic molecular gas, $\gamma$ is equal to $7 / 5$. Equation (3) can be developed either from the conservation of energy or from the conservation of entropy, which turn out to be equivalent for the adiabatic dynamics implied by the assumptions underlying these equations. The Euler equations do describe shocks but only in the limiting sense that they occur as discontinuities. To set the stage for some of the later discussion, it will prove useful to have the energy equation in a conservative form. In this form the energy equation is

$$
\frac{\partial}{\partial t}(\rho \varepsilon)+\frac{\rho u^{2}}{2}=-\nabla \cdot\left(\rho \boldsymbol{u} \varepsilon+\frac{u^{2}}{2}+p \boldsymbol{u}\right) .
$$

There are several other useful forms of the energy equation but this will suffice for our purposes here. 


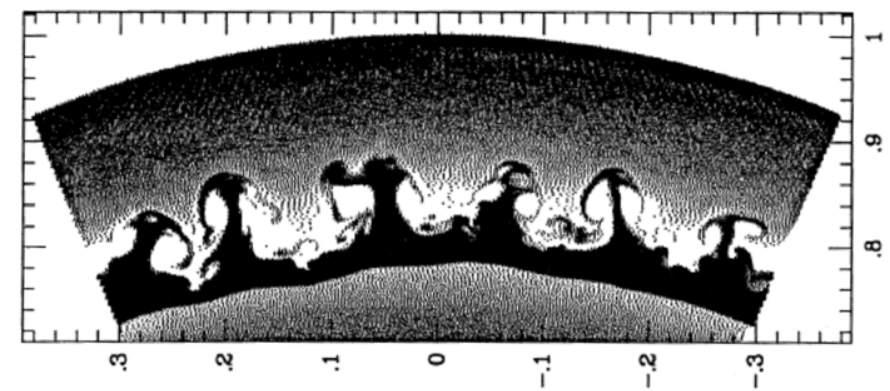

Figure 1. Simulation of a supernova remnant. The greyscale shows density, from [3], reproduced by permission of the AAS. This is a pure-hydrodynamics simulation of an astrophysical system having of order 1 particle $\mathrm{cm}^{-3}$, leading a plasma physicist to wonder about its validity.
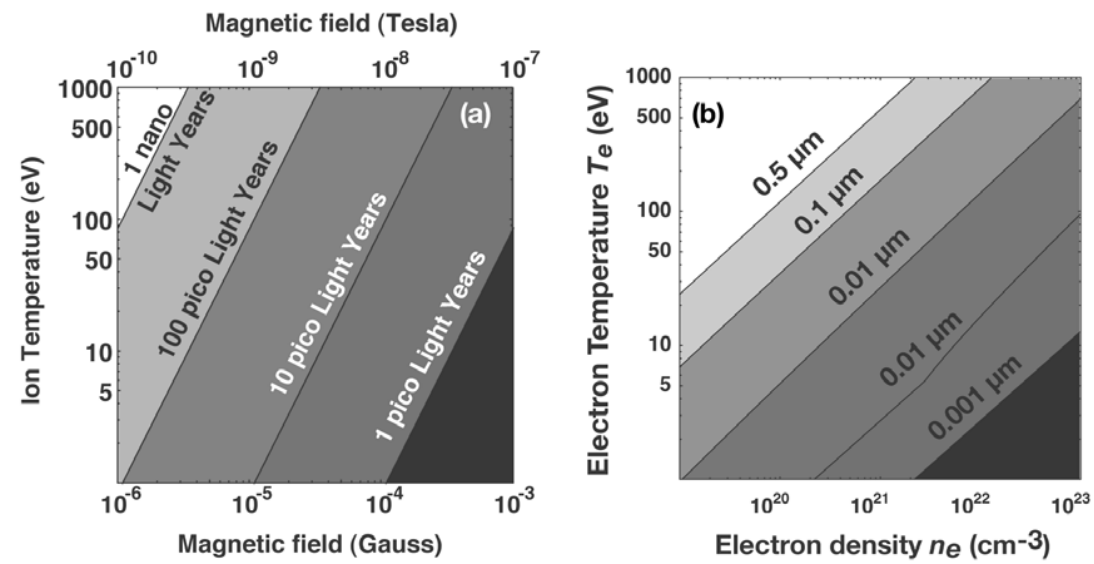

Figure 2. Particle localization in astrophysics and the laboratory. (a) The ion gyroradius in a space of magnetic field and ion temperature. The characteristic pico-light-year scales are at least $10^{9}$ times smaller than structures of interest. (b) The electron mean free path in high-energy-density systems. Characteristic spatial scales in these systems are $100 \mu \mathrm{m}$.

Both inertial fusion and astrophysics involve the dynamics of matter. In both cases, the evolution of structure in the density, velocity and pressure are of great interest. In both cases, shock waves play a central role in changing the properties of matter. This makes it tempting to apply the hydrodynamic equations to them but does not imply that these equations are valid. The first element of validity involves determining whether a fluid model applies at all. This may seem particularly questionable for many astrophysical systems. Figure 1 shows results of a simulation of a supernova remnant [3]. The matter in this simulation is at a density near 1 particle $\mathrm{cm}^{-3}$. The system certainly includes a magnetic field. Collisions play almost no role in the dynamics. Yet the paper reporting these results used a purely hydrodynamic model. Most plasma physicists would initially view this with skepticism. It would seem that, at a minimum, a magnetohydrodynamic (MHD) model would be needed.

Yet in fact a hydrodynamic model is reasonable. The reason is that the magnetic field is not dynamically important but does localize the particles. Figure 2(a) shows why the particles are localized by the magnetic field. Low-density astrophysical systems typically have scales of many lightyears or more. Even SN 1987A, which has been evolving for less than 20 years, has spatial scales that are some fraction of a light-year (and certainly much greater than a millilightyear). Yet the magnetic fields are within a few orders of magnitude of a nanotesla 
and the ion temperatures are typically below $1 \mathrm{keV}$, although they may be larger in recently shocked regions. The result is that the ion orbit is typically below a nanolightyear. In addition, the field lines are not straight over distances that are a great deal larger than an ion orbit. There is instead considerable evidence that the field lines are quite tangled. As a result, it is often completely valid to use a hydrodynamic model to describe low-density astrophysical phenomena. The primary exceptions are when populations of energetic particles become dense enough to affect the hydrodynamics and when radiative cooling becomes significant. In shock-heated astrophysical environments, the ratio of plasma pressure to magnetic-field pressure, $\beta$, is typically very large $(\gg 1)$. This is in sharp contrast to wind-filled circumstellar environments such as the heliosphere, where typically $\beta \sim 1$, or to planetary magnetospheres, within which $\beta<1$.

In the case of inertial fusion and related laboratory experiments, and also within stars, collisions are strong enough to localize the particles. For laboratory systems, this is illustrated in figure $2(b)$, which shows the electron mean free path for a range of parameters relevant to most high-energy-density systems. This includes inertial fusion capsules, although during compression such capsules move off this figure to the right. The typical scales of these laboratory systems are hundreds of microns, with larger values being common in the expanding plasmas corresponding to the upper left portion of this graph. Unstable structures found in systems whose parameters are in the lower right portion of this graph may approach the micron scale. The ions are more localized than the electrons. Thus, collisions localize the particles well enough to use a fluid model for the laboratory system. The magnetic field is typically small enough that $\beta \gg 1$. Exceptions to this do develop at the surfaces of laser-heated targets, in some portions of $z$ pinches, and near the large currents sometimes produced under relativistic conditions, but none of these circumstances are significant in the context of the present discussion. The matter in stars is also collisional, although under some circumstances, such as when heated by the blast wave produced in a supernova explosion, the pressure in stellar plasmas may become dominated by radiation. Since the photons remain well localized within the matter, these radiation-dominated plasmas are well described by the Euler equations with $\gamma=4 / 3$.

Once it is clear that a fluid model is valid, the next question is whether a single-fluid model is adequate or whether additional equations are needed to account for the multiple species in the system. Here we will assert that single-fluid equations are adequate without detailed proof. This amounts to making the claim that neither currents nor charge separation are important, so that the momentum carried by the ions is the dominant factor in the dynamics. If currents were also an important factor, we would need an MHD model, but this is not the case since $\beta \ll 1$. For charge separation to be a factor, the electrons would have to be able to move away from the ions but the magnetic field prevents this in low-density astrophysical environments and collisions prevent it in other environments of interest here.

At this point, having determined that a single-fluid description is adequate, we must ask whether the Euler equations are good enough. In fluid dynamics there are a number of dimensionless parameters that describe the degree to which a hydrodynamic description is valid. Figure 3 shows a more complete fluid equation (for the energy flux), labelling each of the terms in the equation with the corresponding dimensionless parameter. In each case, the dimensionless parameter is the ratio of the hydrodynamic energy flux to the energy flux associated with some non-hydrodynamic effect. Thus, for example, the Reynolds number Re, which is $U L / v$, can be obtained by taking the ratio of the convection of kinetic energy on the left-hand side to the heating by viscosity on the right-hand side. These various terms and parameters are described in detail in Ryutov [11]. Here we show table 1, based on that work, which compares the fluid-dynamics parameters in a supernova and in a supernova remnant with 


$$
\frac{\partial}{\partial t}\left(\rho \varepsilon+\frac{\rho u^{2}}{2}+E_{R}\right)+\nabla \cdot\left[\rho \mathbf{u}\left(\varepsilon+\frac{u^{2}}{2}\right)^{\text {Material Energy }}+p \mathbf{u}\right]=
$$

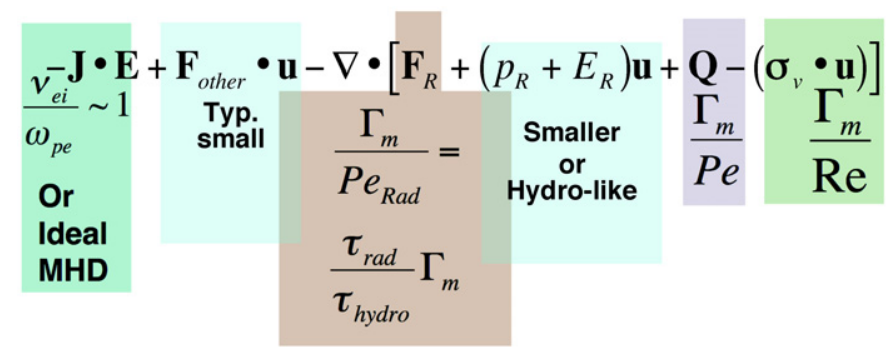

Figure 3. A general single-fluid energy equation including effects of joule heating, radiation, heat conduction and viscosity. The labelling shows the various fluid dynamics parameters that determine whether terms can be dropped. Here the radiation pressure, energy density, and flux are $p_{R}, E_{R}$ and $\mathbf{F}_{\mathrm{R}}$, respectively, $\mathbf{J}$ is the current density, $\mathbf{E}$ is the electric field, $\mathbf{F}_{\text {other }}$ is other forces, $\mathbf{Q}$ is the heat flux, $\boldsymbol{\tau}_{\tau}$, is the viscosity tensor, $\tau_{\mathrm{ei}}$ is the electron-ion collision rate, $\tau_{\mathrm{pe}}$ is the plasma frequency, and the timescales for hydrodynamic evolution and radiative cooling are $\tau_{\text {hydro }}$ and $\tau_{\text {rad }}$, respectively.

Table 1. Fluid dynamics parameters in various astrophysical and laboratory environments (based on [11]).

\begin{tabular}{lllll}
\hline $\begin{array}{l}\text { Derived } \\
\text { parameters }\end{array}$ & Supernova & Lab & $\begin{array}{l}\text { Supernova } \\
\text { remnant }\end{array}$ & $\begin{array}{l}\text { Low-density } \\
\text { lab }\end{array}$ \\
\hline$v /(p / r)^{1 / 2}$ & 2.2 & 2.3 & 3.0 & 2.9 \\
Localization & $3 \times 10^{13}$ & $2 \times 10^{5}$ & $3 \times 10^{7}$ & $1 \times 10^{6}$ \\
Reynolds \# Re & $2 \times 10^{11}$ & $2 \times 10^{5}$ & $6 \times 10^{8}$ & $7 \times 10^{6}$ \\
Peclet \# Pe & $1 \times 10^{13}$ & $4 \times 10^{3}$ & $1 \times 10^{7}$ & $1 \times 10^{4}$ \\
Radiation $P e_{\mathrm{Rad}}$ & $2 \times 10^{15}$ & $5 \times 10^{9}$ & $3 \times 10^{6}$ & 380 \\
\hline
\end{tabular}

their values in corresponding high-energy-density experiments. Inertial fusion experiments would not be very different from the other laboratory experiments in this regard.

As a final remark on this subject, once it is clear that two types of systems obey the Euler equations, scaling between them is fairly straightforward. If one can create structures of similar shapes, with appropriate boundary conditions, one can obtain, for example, in the laboratory, systems whose dynamics is substantially identical to that in an astrophysical system. This is the topic of a sequence of papers by Ryutov and collaborators [11-13].

\section{Theory of bouyancy-driven instabilities and Rayleigh-Taylor}

The fundamental buoyant driving force for Rayleigh-Taylor instabilities is readily understood by thinking of a fluid subject to gravitational acceleration, the opposite of which defines height $(z)$ in the system, as illustrated in figure 4 . The fluid adjusts to equalize the pressure at any given elevation, so at that elevation the average density determines the pressure gradient. Yet any local element of fluid experiences a force proportional to its own density. If the local density in a fluid element is below the average density at that elevation, then that fluid element experiences an upward buoyant force (and vice versa). The net force experienced by the fluid 


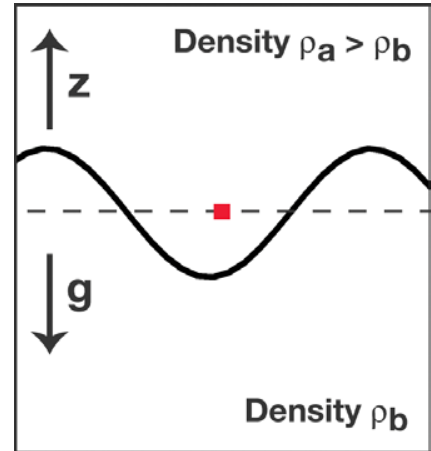

Figure 4. The basic drive of the Rayleigh-Taylor instability is the difference between the average density and the local density along a surface of constant potential energy.

element is $(\langle\rho\rangle-\rho) g$, where $\rho$ is the density, \langle\rangle denotes the average at some elevation and $g$ is the gravitational acceleration. The result is that whenever the density gradient is opposed to the pressure gradient, small ripples will unstably grow.

A simple example of a buoyancy-driven instability that is not Rayleigh-Taylor occurs at a constant density when the temperature decreases with increasing elevation. Then any fluid element that rises slightly will expand, decrease in density and experience an upward buoyant force (and again vice versa). This leads, for example, to convective mixing in the atmosphere of the Earth.

The general statement that bridges these two examples and cases without gravity is that any system in which the entropy gradient is parallel to the pressure gradient is subject to a buoyancy-driven instability. The term Rayleigh-Taylor instability is used specifically for the case of a density gradient (or a discontinuous change in density). The more general case can be worked out as follows.

Consider a planar system having initial gradients in the pressure, entropy and other variables and let these gradients define the $z$ direction. Also assume that any second derivatives of the initial values of these quantities are negligible except that there may be, at some surface of interest, a jump in entropy (and thus density and temperature). The pressure is necessarily continuous across any surface (and everywhere except at shocks). Such surfaces are certainly relevant in inertial fusion and at times are of interest as well in astrophysics. Suppose that small-amplitude fluctuations develop, which are adiabatic (thus conserving entropy) and incompressible (thus expending no energy on compression and perhaps growing most rapidly as a result). Designate the initial, steady quantities using the subscript ' 0 ' and the fluctuating quantities using the subscript ' 1 '. Use $p, \rho, s$ and $\boldsymbol{u}$ for pressure, density, entropy and velocity, respectively. In the frame of reference of an external observer the fluid accelerates with acceleration $\boldsymbol{a}=-\nabla p_{0} / \rho_{0}$. In the accelerating frame of reference of a surface within the fluid, the local acceleration has the opposite sign, being $\boldsymbol{g}=\nabla p_{0} / \rho_{0}$. Recognizing this is one key element in connecting various literature on Rayleigh-Taylor.

Writing the linearized equations that describe the behaviour of the system in the frame of reference of the interface, one has entropy conservation,

$$
\frac{\partial}{\partial t} s_{1}=-\boldsymbol{u}_{1} \cdot \nabla s_{0}
$$

incompressibility of the fluctuations,

$$
\nabla \cdot \boldsymbol{u}_{1}=0
$$


and the linearized equation of motion in the frame of the interface, including the acceleration $\nabla p_{0} / \rho_{0}$, as

$$
\rho_{0} \frac{\partial}{\partial t} \boldsymbol{u}_{1}=-\nabla p_{1}+\frac{\nabla p_{0}}{\rho_{0}} \rho_{1}
$$

in which the average pressure-gradient-driven acceleration has cancelled out the average 'gravitational' acceleration, $\nabla p_{0} / \rho_{0}$. We will assume this ratio to be constant, or equivalently that its constant value designates the acceleration of the frame we are working in. For now we want to use entropy and pressure as the thermodynamic variables, so we write

$$
\rho_{1}=\left(\frac{\partial \rho_{0}}{\partial s_{0}}\right)_{p} s_{1}+\left(\frac{\partial \rho_{0}}{\partial p_{0}}\right)_{s} p_{1},
$$

in which the second term introduces no new qualitative features and is negligible in magnitude for all conditions of interest (because $p_{1} \ll \rho_{0} c_{\mathrm{s}}^{2}$, where $c_{\mathrm{s}}$ is the isentropic sound speed); so we ignore it. Equation (7) then becomes

$$
\rho_{0} \frac{\partial}{\partial t} \boldsymbol{u}_{1}=-\nabla p_{1}+\frac{\nabla p_{0}}{\rho_{0}}\left(\frac{\partial \rho_{0}}{\partial s_{0}}\right)_{p} s_{1} .
$$

We assume that the fluctuating quantities are proportional to $\exp \left[\mathrm{i} k_{x} x+n t\right]$, where $n$ is the growth rate and $k_{x}$ is the component of the wave vector in the $x$ direction. We identify the components $\boldsymbol{u}_{1}$ by $\boldsymbol{u}_{1}=(u, v, w)$, noting that there are no driving terms for transverse waves so we can take $v=0$. Equation (6) tells us that $\mathrm{i} k_{x} u=-\partial w / \partial z$ so the $x$ component of equation (9) becomes

$$
\rho_{0} n \frac{\partial w}{\partial z}=-k_{x}^{2} p_{1}
$$

Equation (5) leads us to take the derivative in $t$ of the $z$ component of equation (9) and noting that $-\boldsymbol{u}_{1} \cdot \nabla s_{0}=-w \partial s_{0} / \partial z$ we find

$$
\rho_{0} n^{2} w=-n \frac{\partial p_{1}}{\partial z}-\frac{1}{\rho_{0}} \frac{\partial p_{0}}{\partial z}\left(\frac{\partial \rho_{0}}{\partial s_{0}}\right)_{p} \frac{\partial s_{0}}{\partial z} w .
$$

Solving equation (10) for $p_{1}$, taking the derivative in $z$ and substituting into equation (11) gives

$$
\rho_{0} n^{2} w=\frac{n^{2}}{k_{x}^{2}}\left(\rho_{0} \frac{\partial^{2} w}{\partial z^{2}}+\frac{\partial w}{\partial z} \frac{\partial \rho_{0}}{\partial z}\right)-\frac{1}{\rho_{0}} \frac{\partial p_{0}}{\partial z}\left(\frac{\partial \rho_{0}}{\partial s_{0}}\right)_{p} \frac{\partial s_{0}}{\partial z} w
$$

in which we have taken $\partial \rho_{0} / \partial z$ to be a first order quantity. (Various derivations in the literature, such as that in the fluid dynamics volume of Landau and Lifshitz, assume this quantity to be negligible.) Defining

$$
\omega_{\mathrm{s}}^{2}=\frac{1}{\rho_{0}^{2}} \frac{\partial p_{0}}{\partial z}\left(\frac{\partial \rho_{0}}{\partial s_{0}}\right)_{p} \frac{\partial s_{0}}{\partial z}
$$

for temporary convenience and taking $(1 / L)=\partial\left(\ln \rho_{0}\right) / \partial z$, we can rewrite this as

$$
\left(1+\frac{\omega_{\mathrm{s}}^{2}}{n^{2}}\right) k_{x}^{2} w-\left(\frac{1}{L}\right) \frac{\partial w}{\partial z}-\frac{\partial^{2} w}{\partial z^{2}}=0 .
$$

In the limit of $L$ so large that the second term can be dropped, the solution of this equation is plane waves with

$$
n^{2}=-\omega_{\mathrm{s}}^{2} \sin ^{2} \theta
$$

where $\theta$ is the angle between the $z$ axis and $\boldsymbol{k}$. If one now examines equation (13) and realizes that $\left(\partial \rho_{0} / \partial s_{0}\right)_{p}<0$, then one can see that the fluctuations are unstable if the pressure and 
entropy gradients are parallel. This is sometimes called the entropy mode. The important general consequence is that instability occurs when there is an entropy gradient even in the absence of a density gradient.

For finite $L$, the solution of equation (14) is

$$
\begin{aligned}
w=C_{1} \exp [ & \left.\left(\frac{z}{2 L}\right)\left(\sqrt{1+4 k_{x}^{2} L^{2}+4 \frac{k_{x}^{2} L^{2} \omega_{\mathrm{s}}^{2}}{n^{2}}}-1\right)\right]+C_{2} \exp \left[\left(-\frac{z}{2 L}\right)\right. \\
& \left.\times\left(\sqrt{1+4 k_{x}^{2} L^{2}+4 \frac{k_{x}^{2} L^{2} \omega_{\mathrm{s}}^{2}}{n^{2}}}+1\right)\right]
\end{aligned}
$$

and one must specify boundary conditions for the unstable mode to get a solution.

Before specifying the boundary conditions it is useful to change thermodynamic variables and to express the entropy as a function of pressure and density, so

$$
\begin{gathered}
\left(\frac{1}{\rho_{0}} \frac{\partial p_{0}}{\partial z} \frac{\partial \rho_{0}}{\partial s_{0}}\right) \frac{\partial s_{0}}{\partial z}=\frac{1}{\rho_{0}} \frac{\partial p_{0}}{\partial z}\left(\frac{\partial \rho_{0}}{\partial s_{0}}\right)_{p}\left[\left(\frac{\partial s_{0}}{\partial p_{0}}\right)_{\rho}\left(\frac{\partial p_{0}}{\partial z}+\frac{\partial s_{0}}{\partial \rho_{0}}\right)_{p} \frac{\partial \rho_{0}}{\partial z}\right] \\
=\frac{1}{\rho_{0}} \frac{\partial p_{0}}{\partial z}\left[-\frac{1}{c_{\mathrm{s}}^{2}} \frac{\partial p_{0}}{\partial z}+\frac{\partial \rho_{0}}{\partial z}\right]=\rho_{0} \omega_{\mathrm{s}}^{2},
\end{gathered}
$$

in which we have used the result from differential calculus that

$$
\left(\frac{\partial \rho_{0}}{\partial s_{0}}\right)_{p}\left(\frac{\partial s_{0}}{\partial p_{0}}\right)_{\rho}=-\left(\frac{\partial \rho_{0}}{\partial p_{0}}\right)_{s} .
$$

Note that in equation (16) for very large $k_{x} L$ and a pressure gradient that is correspondingly small, the argument of the exponents goes to $\pm k_{x} z$. This, for example, is the spatial behaviour in the case of a density jump in an otherwise uniform medium.

To specify the boundary conditions one must choose a more specific case. We consider here the simple case that the mode must decay at \pm infinity. The two solutions for the velocity must match at the surface of interest (the location of the largest amplitude or the density jump). Choosing this to be $z=0$ implies that $C_{1}=C_{2}$. We can find the boundary condition that gives $n$ by integrating equation (11) across the surface after substituting from equation (17), subtracting eqution (10) across the surface and eliminating $p_{1}$. This gives a relation between the derivatives of the velocity in the two regions. We label the quantities evaluated as the surface is approached from above with the subscript ' $a$ ' and quantities evaluated as the surface is approached from below with the subscript ' $b$ '. All of this gives us

$$
\rho_{\mathrm{b}} \frac{\partial w_{\mathrm{b}}}{\partial z}-\rho_{\mathrm{a}} \frac{\partial w_{\mathrm{a}}}{\partial z}=\frac{k_{x}^{2}}{n^{2}} \frac{\left|\nabla p_{0}\right|}{\rho_{0}}\left(\rho_{\mathrm{a}}-\rho_{\mathrm{b}}\right) C_{1} .
$$

In the simplest case when there is no density jump at the surface, equations (16) and (19) give for the growth rate the solution

$$
n^{2}=-\omega_{\mathrm{s}}^{2} \frac{4 k_{x}^{2} L^{2}}{1+4 k_{x}^{2} L^{2}} .
$$

One again finds growth when $\omega_{\mathrm{s}}^{2}$ is negative, corresponding to parallel pressure and entropy gradients. But now the growth rate is reduced under any condition except $k_{x} L \rightarrow \infty$.

When there is a jump at the interface, it helps to write

$$
\omega_{\mathrm{s}}^{2}=-\frac{1}{\rho_{0}} \frac{\partial p_{0}}{\partial z}\left[\frac{1}{\rho_{0} c_{\mathrm{s}}^{2}} \frac{\partial p_{0}}{\partial z}-\frac{1}{\rho_{0}} \frac{\partial \rho_{0}}{\partial z}\right]=-g k_{\mathrm{p}} .
$$

Note that $\omega_{\mathrm{s}}$ is a property of the large-scale medium and not of the interface. Also note that when the pressure gradient is comparatively negligible, $\left|k_{\mathrm{p}}\right|=1 / L$, with the sign determined 
by whether the density and pressure gradients are parallel or opposed. With this notation, we can solve equations (16) and (19) to obtain

$n^{2}=g k_{x}\left(\frac{2 k_{x} L}{1+4 k_{x}^{2} L^{2}-A^{2}}\right)\left[k_{\mathrm{p}} L-A^{2}+\sqrt{k_{\mathrm{p}}^{2} L^{2}+A^{2}\left(1+4 k_{x}^{2} L^{2}-2 k_{\mathrm{p}} L\right)}\right]$,

where we have as usual defined an Atwood number $A=\left(\rho_{\mathrm{a}}-\rho_{\mathrm{b}}\right) /\left(\rho_{\mathrm{a}}+\rho_{\mathrm{b}}\right)$.

Equation (22) has all the limits one finds in various literature except for the case of a localized density ramp matching uniform regions. On the one hand, if there is a density jump, if the pressure gradient can be ignored and if the density gradient opposes the pressure gradient, so $k_{\mathrm{p}}=1 / L$, when as $k_{x} L \rightarrow \infty$ one finds

$$
n=\sqrt{A k_{x} g},
$$

which is the result most familiar to laboratory scientists, but may also be relevant to some astrophysical systems. One example is a young supernova remnant, in which the contact surface between the expanding stellar ejecta and the shocked circumstellar matter has a very abrupt jump in density [14].

On the other hand, if the pressure gradient can be ignored the density gradient opposes the pressure gradient, so $k_{\mathrm{p}}=1 / L$, and there is no local density jump, so $A=0$, then

$$
n=\sqrt{k_{x} g}\left(\sqrt{\frac{4 k_{x} L}{1+4 k_{x}^{2} L^{2}}}\right),
$$

which is relevant to short-wavelength modes in both inertial fusion and astrophysics.

Another standard case arises if we take $A=0$, define $L_{\mathrm{p}}$ by $\left(1 / L_{\mathrm{p}}\right)=\partial\left(\ln p_{0}\right) / \partial z$ and assume a polytropic gas so that $c_{\mathrm{s}}^{2}=\gamma p_{0} / \rho_{0}$ and evaluate $g$ in terms of $c_{\mathrm{s}}$. This gives

$$
n^{2}=\frac{c_{\mathrm{s}}^{2}}{\gamma}\left(\frac{4 k_{x}^{2} L^{2}}{1+4 k_{x}^{2} L^{2}}\right)\left[\frac{1}{\gamma L_{\mathrm{p}}^{2}}-\frac{1}{L_{\mathrm{p}} L}\right] .
$$

This, is in the limit of large $k_{x} L$ so that the term in curved brackets equals unity, most familiar in the astrophysics literature. One can see that a pressure gradient is inherently destabilizing but that a density gradient is only destabilizing if it opposes the pressure gradient. When the scale length of the density profile is large and it makes sense to consider modes for which $k_{x} L \gg 1$, the growth rate loses any dependence on $k$. It becomes of the order of the sound speed divided by the characteristic scale of the system (formally $\sqrt{L_{\mathrm{p}} L}$ ). This may seem strange to those whose focus has long been in regimes where the Rayleigh-Taylor growth rate is $\sim \sqrt{k g}$ and thus depends on $k$ but even so this remains fundamentally the same buoyancy-driven instability.

The applications of equation (25) include all the regimes of buoyancy driven instabilities that one encounters in extended gradients in inertial fusion, in other high-energy-density experiments and in astrophysics. The case known as the entropy mode arises in inertial fusion during the implosion of the fusion capsule. Current designs emphasize the production of high entropy in the outer layers of a capsule, where the pressure is also higher. This creates parallel entropy and pressure gradients despite the fact that the density is fairly uniform. As a result, the term involving $L_{\mathrm{p}}^{2}$ in equation (21) produces unstable growth. This must be evaluated as part of any complete assessment of an inertial fusion capsule design [15]. One astrophysical application would be the heating of a region of uniform density that is near a hot source such as a newly formed star, so that the heating (and consequent pressure) is non-uniform. A range of convective phenomena in the Earth's atmosphere also arise by the same mechanism, as the density in the Earth's atmosphere varies more slowly with altitude than the temperature does. 

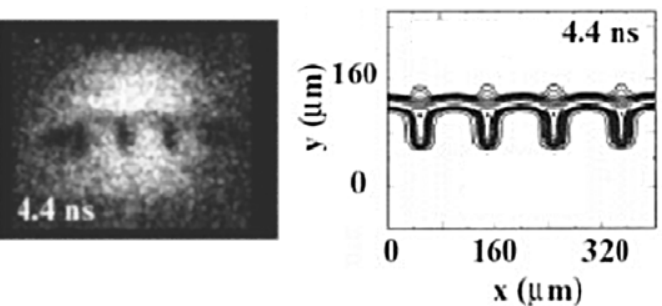

Figure 5. Experimental x-ray radiograph $(a)$ and simulation results showing constant-density contours $(b)$ for the ablative Rayleigh Taylor instability, reprinted with permission from [16], American Institute of Physics.

\section{Examples of Rayleigh Taylor in the laboratory and in astrophysics}

Examples of the Rayleigh-Taylor instability abound in both laboratory and astrophysical systems. In inertial fusion, a fuel capsule must be accelerated to a high inward velocity and then decelerated to rest in order to fully compress the fuel. The acceleration is produced by ablation of the material and so is described as ablatively driven. Rayleigh Taylor growth must be kept small in ICF, otherwise the capsule may break up or the imploded fuel may become too convoluted to burn well. A simple calculation shows that this is not trivial. We consider the growth of a mode having $k \sim 2 \pi / \Delta R$, where $\Delta R$ is the thickness of the capsule during acceleration, because such a mode is the most disruptive. Making reasonable assumptions that the interface where the lower-density plasma pushes on the dense fuel is abrupt and that the density jump is large enough that $A \sim 1$, we use equation (23) for the growth rate. To find the number of e-foldings of growth we need to know the acceleration, $g$, and the implosion time, $t$. On the simple assumption that the capsule is accelerated for the first half of the implosion and is decelerated for the second half, we relate $t$ and $g$ as

$$
\frac{R}{2}=\left(\frac{1}{2}\right) g\left(\frac{t}{2}\right)^{2},
$$

in which $R$ is the radius of the capsule when the acceleration starts. Solving this equation for $t$, we find

$$
n t=\sqrt{\frac{8 \pi R}{\Delta R}} .
$$

This is of order 50 for typical values of $R / \Delta \mathrm{R}$. However, for realistic initial conditions one requires that $n t$ be less than about 10 . This is one of the major reasons that inertial fusion is not trivial.

Fortunately, in the presence of gradients and ablation (which we did not consider in the above derivation) the growth rate is approximately given by

$$
n \approx \sqrt{\frac{k g}{1+k L}}-\beta k v_{\text {ablation }}
$$

in which the velocity at which material is removed from the ablation surface where the pressure reaches its maximum is $v_{\text {ablation }}$, and $\beta$ is a coefficient of order 1 . The combination of gradient scale length and ablation appears to be sufficient to allow inertial fusion to succeed, but only if serious attention is paid, in the design and the technical tolerances, to minimization of the Rayleigh-Taylor growth.

Many experiments have explored this ablative regime of Rayleigh Taylor, in some cases to levels of growth far beyond those allowable for ICF. Figure 5 shows both imaging data 


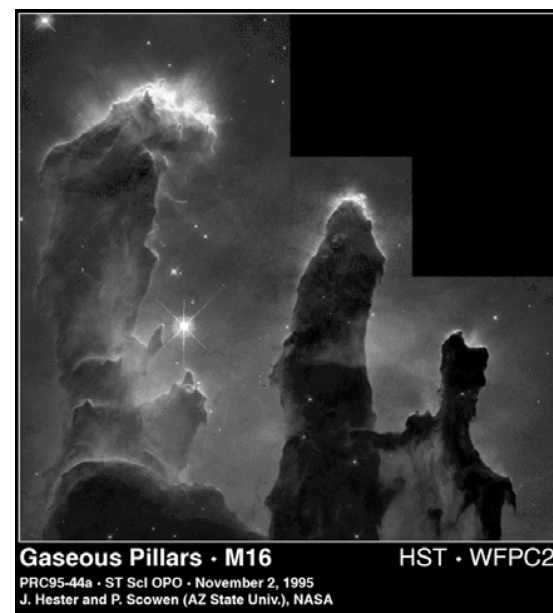

Figure 6. Image of the Eagle Nebula, from the WFPC2 camera on the Hubble Space Telescope. Credit: Jeff Hester and Paul Scowen (Arizona State University) and NASA.

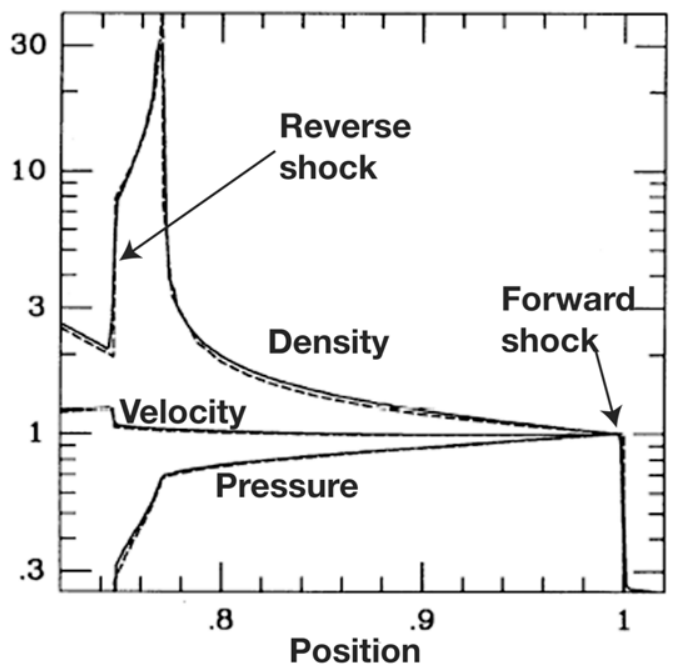

Figure 7. Radial profiles of parameters in a 1D model of a supernova remnant, from [3], reproduced by permission of the AAS.

(using x-ray radiography) and a simulation from one such case [16]. In astrophysical systems affected by intense radiation, such as star-forming regions where there are many young, very hot stars, ablative Rayleigh Taylor instabilities are also possible. This is one explanation of the well-known structures in the Eagle Nebula, shown in figure 6.

Another circumstance that produces Rayleigh Taylor instabilities is the acceleration of lower density material by a flowing plasma. One such case occurs when the flowing ejecta from a supernova encounter the material in the circumstellar medium. The consequent structure is illustrated in figure 7. Overall, the momentum from the ejecta sustains a forward shock, driven into the circumstellar medium. In more detail, structure develops where the two media interact. The stellar ejecta enter from the left, where they encounter the reverse shock that has arisen out of the interaction. This creates a zone of shocked ejecta, ending at a contact surface 


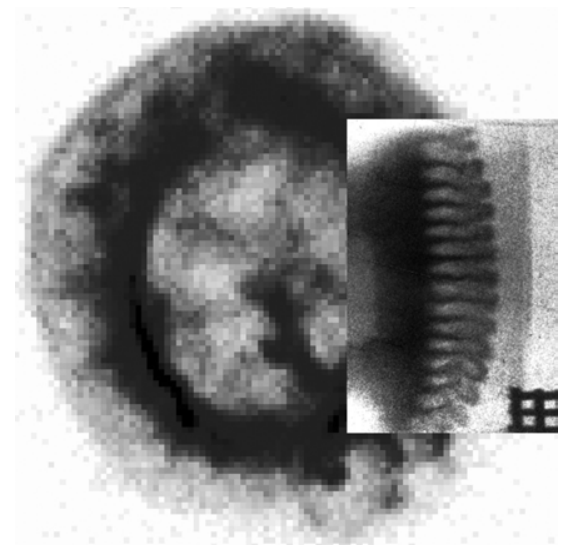

Figure 8. Composite image of E0102 from radio to $\mathrm{x}$-ray. The inset shows data from experiments involving the author. For the E0102 image, credit: Jack Hughes (Rutgers), x-ray (NASA/CXC/SAO); optical (NASA/HST); radio: (ATNF/ ATCA) http://antwrp.gsfc.nasa.gov/apod/ap000414.html.

where the density drops abruptly to the level of the shocked circumstellar matter. The steady accumulation of shocked circumstellar matter is manifested by the pressure gradient also seen in the figure, causing a deceleration of the layer between the two shocks and creating a zone that is Rayleigh-Taylor unstable. The consequences, in simulation, were shown in figure 1.

Figure 8 shows data from astrophysical and laboratory systems having similar dynamics. Most of figure 8 shows a composite image of a nearby supernova remnant, E0102, which has been imaged in several frequency ranges from the radio to the $\mathrm{x}$-ray. The greyscale represents a convolution of composition and temperature. The outer rim of the image is interpreted to be the forward shock described above. The transition from the material just behind this shock to the next (darker) layer of material is modulated. The observed structure is quite possibly due to Rayleigh-Taylor instabilities at the contact surface described just above. The next transition from dark to light, at smaller radius, may be the reverse shock. The inset in this figure shows results from a laboratory experiment in planar geometry, described below. As in the supernova remnant, the laboratory experiment develops forward and reverse shocks. It also develops large-amplitude instability growth at the contact surface (in this case seeded by an initial perturbation).

The unstable structures in figure 8 were produced by a (planar) blast wave. Blast waves are a common source of instability growth in supernovae and in other astrophysical systems as well as in laboratory experiments, and so their evolution merits some discussion, with reference to figure $9(a)$. A blast wave is produced when a brief energy source drives a shock through a system and then ceases. After the energy source ends, the surface where the pressure was applied expands, creating a flow of material away from the shock and driving a disturbance known as a rarefaction wave towards the shock. The blast wave forms when the rarefaction wave overtakes the shock. The blast wave has an initial shock followed by a region of decreasing pressure, density and velocity. The structure does not change further except that the shock grows weaker with time. (In some subfields, the term 'blast wave' is reserved for spherical systems and more convoluted language is used for other cases, but the description above is independent of geometry.)

Figure $9(b)$ shows how a blast wave that reaches an interface can evolve into a structure that is similar to that shown in figure 7. After the leading edge of the blast wave reaches an interface where the density drops, the density develops a stepped structure. The leading 

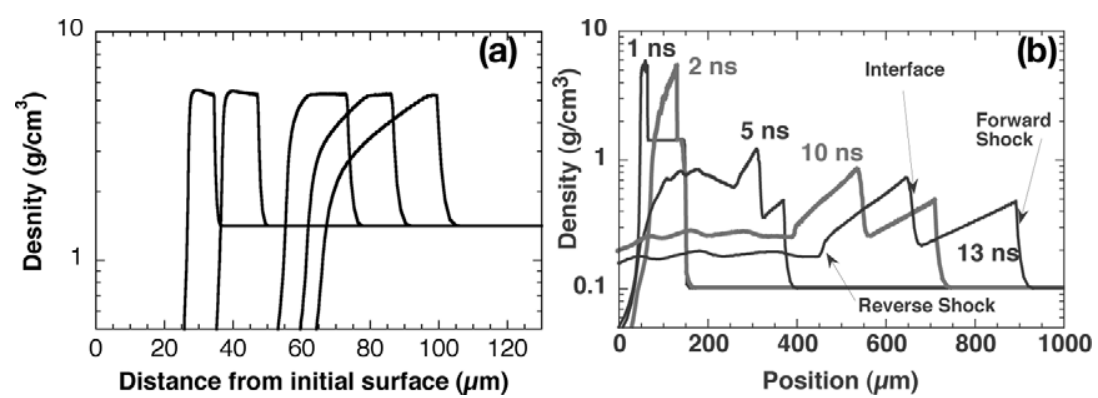

Figure 9. Development of a blast wave, from [58] (a) and dynamics when a blast wave encounters an interface with decreasing density $(b)$, from [58].

edge remains a shock wave, but the shock is unable to compress the lower-density material to the density of the material from the first layer (because the density ratio that a single strong shock can produce is limited). The result is that there is a forward shock in the low-density material a layer of shocked low-density material and then a contact surface where the density increases, at the boundary between the material from the two initial layers. This second jump in density at the contact surface is not a shock; the pressure is continuous across it. As the low-density material and the inflowing material from the initial blast wave both accumulate, the shocked layer slows down and a reverse shock develops in the inflowing material. In addition, a pressure gradient develops that opposes the density gradient at the contact surface, creating a Rayleigh-Taylor unstable state.

This type of evolution occurs, for example, within a core-collapse supernova. A corecollapse supernova is initiated by the collapse of the iron core in a massive star. This happens when the core has accumulated enough mass to enable gravity to overcome the degeneracy pressure of the electrons, collapsing the atoms in the core into neutrons [1]. The resulting dynamics launches a blast wave through the rest of the star, which blows the star apart. In the process, the blast wave encounters several interfaces where the mass density decreases comparatively abruptly. This creates the dynamics described with reference to figure 9 , leading to Rayleigh-Taylor instabilities.

The ultimate impact of these instabilities is poorly understood for several reasons. As they cannot be directly observed, knowledge of the behaviour can only be inferred from simulations and scaled experiments, constrained by observations made after the explosion. The initial conditions for the explosion are becoming more clear, but are not yet well known. Fully 3D simulations of the explosion are not yet practical, and there are known to be differences between the $2 \mathrm{D}$ simulations that exist and the real $3 \mathrm{D}$ behaviour. Even so, the $2 \mathrm{D}$ simulations show the evolution of highly structured interfaces and substantial penetration of the less dense layers by material from the denser layers [17-21]. 3D experiments are possible but at present only for a single stellar interface and in a planar geometry. Such experiments remain well scaled until the interface in the experiment 'discovers' that it is not spherical when it is affected by disturbances from the edges. The major facilities under construction will soon enable experiments to address the effects due to multiple interfaces in a diverging geometry, moving at that point well beyond the level of detail at which simulations can be performed.

The technical approach to conducting a blast wave-driven instability experiment is shown in figure 10. A number of laser beams (typically 10 on the Omega facility [22]) strike a surface, which is surrounded radially by a large disc to absorb the laser light at the edges of the laser beam. The laser beams ablate the irradiated surface, driving a shock wave into a dense layer (often of plastic). After the laser beams end, a blast wave forms. The thickness of the dense 


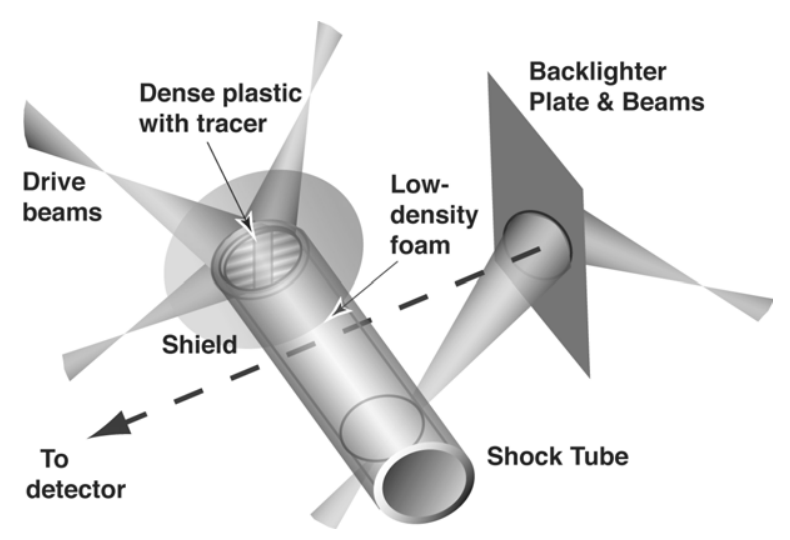

Figure 10. Schematic drawing of the target and the experiment producing a blast wave using an intense laser, from [33].

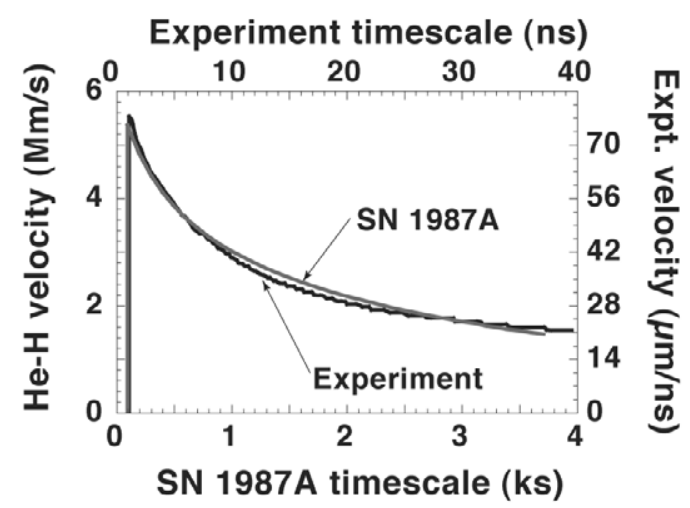

Figure 11. Interface velocity in SN1987A and an experiment, from computer simulations (adapted from [28]).

layer is chosen to tailor the blast wave. Embedded in the dense layer and matched in density with it is a tracer strip, including an element such as $\mathrm{Br}$ that is a strong absorber of the diagnostic $\mathrm{x}$-rays. A lower density material, such as an aerogel foam, follows. The target components are contained within a low- $Z$ shock tube. Other laser beams strike a material (e.g. V) chosen to produce energetic $x$-rays to produce an image of the target.

An extended sequence of experiments has developed the technique just described and obtained data intended specifically to be relevant to the evolution of the $\mathrm{H}-\mathrm{He}$ interface in supernova SN 1987A [23-36]. Figure 11 compares the interface velocity produced in one of these experiments, from a simulation confirmed by observations, with the interface velocity in the supernova from simulations. In this and other respects this is a wellscaled experiment [11]. Later experiments have developed improved technologies and have progressed from barely being able to observe the spikes produced by the instability to studying their long-term behaviour and exploring various aspects of the system such as divergence and interface coupling. These papers and others [37-43] have included extensive comparisons with simulations. Related experiments were also undertaken by a French group $[44,45]$. 


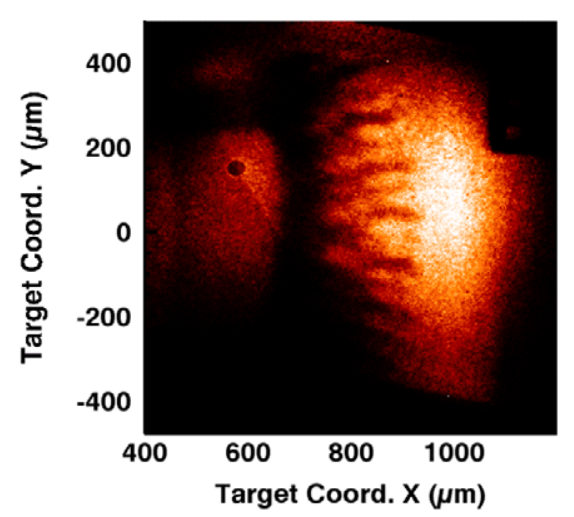

Figure 12. Highly structured interface, with extended spikes of dense material, from [33].

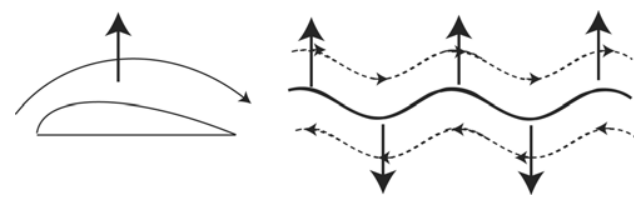

Figure 13. The lift produced by variations in fluid velocity, familiar from airplace wings (as on the left) is also responsible for the unstable growth of ripples in flows with velocity shear, by the Kelvin-Helmholtz instability (on the right).

Figure 12 shows an image from one experiment [33]. The image is an x-ray radiograph, taken at $13 \mathrm{~ns}$ in the experiment. Darker regions in the image show smaller $\mathrm{x}$-ray intensity, produced primarily by absorption in the $\mathrm{Br}$ dopant that was included in a tracer within the denser material. The instability has produced extensive, 3D structures in which spikes of dense material are penetrating deeply into the less-dense material. The initial conditions in this case included a 3D mode in an egg crate pattern in additon to a longer-wavelength squarewave perturbation. Later in this experiment, the dense material appears to penetrate all the way to the forward shock. The experiments just mentioned set the stage for experiments that will proceed to study how the instability develops in well-scaled experiments that progressively employ more complex, 3D and ultimately realistic initial conditions.

\section{Instabilities driven by lift}

The second primary force that leads to hydrodynamic instabilities is lift. The classic lift-driven instability is the Kelvin Helmholtz, illustrated in figure 13. An airplace wing, shown to the left of this figure, produces lift because of the Bernoulli effect, by which a more rapidly flowing fluid has a reduced pressure. This also occurs across any ripples in an interface where there is a flow-velocity shear (a gradient in velocity perpendicular to the direction of flow). The lift that is generated causes the ripples to grow, which produces rippled structures in many circumstances.

The linear theory of this instability involves a straightforward application of the Euler equations combined with the equation that guarantees continuity of the interface,

$$
\frac{\partial \delta \boldsymbol{x}_{\mathrm{s}}}{\partial t}+\boldsymbol{u} \cdot \nabla \delta \boldsymbol{x}_{\mathrm{s}}=\boldsymbol{u}_{\mathrm{s}}
$$




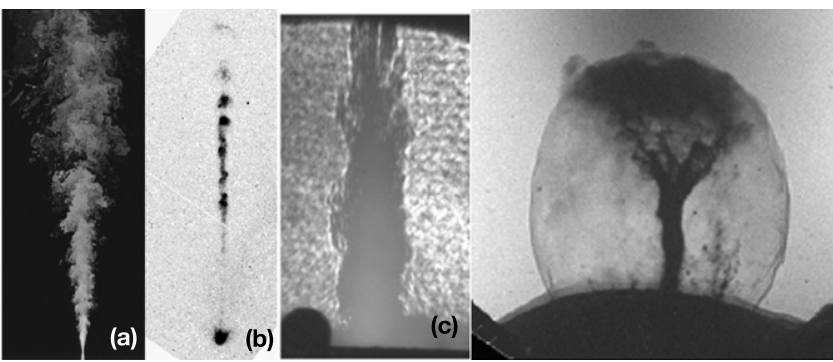

Figure 14. Comparison of various jets. (a) Laser-induced fluorescence image of round jet in water, at $R e=10^{4}$, from [46], with permission. (b) The jet in HH34, obtained by WFPC 2 on the Hubble space telescope. Credit: Burrows, Hester, Morse and NASA. (c) Jet produced in a $z$ pinch, by Lebedev et al with permission (d) Jet produced in a laser experiments by J Foster and the Omega Jet Team.

in which the interface position and velocity are $\boldsymbol{x}_{\mathrm{s}}$ and $\boldsymbol{u}_{\mathrm{s}}$, respectively. The resulting exponential growth rate, $n$, is

$$
n=-\mathrm{i} k_{x} \frac{A}{2} \Delta U+\frac{k_{x} \Delta U}{2} \frac{2 \sqrt{\rho_{\mathrm{a}} \rho_{\mathrm{b}}}}{\left(\rho_{\mathrm{a}}+\rho_{\mathrm{b}}\right)} .
$$

Here the first term causes the ripples to propagate but only if there is a density jump at the interface (so $A \neq 0$ ). The simple Kelvin-Helmholtz instability has a growth rate for all wavelengths without limit, increasing as the wavelength decreases. If this were the entire story then all shear flows would initially develop small-scale structures, followed by larger scale structures only later. This does not occur in practice. One reason is that viscous diffusion creates a finite scale length in the velocity shear, increasing with time. Designating this scale length by $L_{\mathrm{u}}$, one finds that modes with $k_{x}$ larger than about $1 / L_{\mathrm{u}}$ are stabilized.

One of the most dramatic examples of Kelvin Helmholtz instabilities in low energy-density laboratory experiments is the evolution of jets in gasses. Figure 14 compares this evolution with one astrophysical and two high-energy-density examples. The jet in gas develops a KelvinHelmholtz structure, then quickly develops modulations around the jet and then develops a turbulent structure. The astrophysical jet does not do this, nor does the jet in the $z$ pinch or in the laser. Yet the shear in velocity $\Delta U$ in this and many similar laser experiments is $>10 \mathrm{~km} \mathrm{~s}^{-1}$, while the scale on which we could see the instability is $20 \mu \mathrm{m}$. On this scale the instability growth rate is of the order of a nanosecond. It seems likely that all the more-stable systems (figures $14(b)-(d)$ ) develop a gentle radial gradient in velocity which quenches the instability. (At the time of the image in figure $14(d)$, the trunk of the jet is quite cool and surface tension may play a role, but Kelvin-Helmohltz is also not observed earlier in this experiment when the jet is warmer.) One of the major differences among simulations is the degree to which they develop small-scale, Kelvin-Helmholtz structures along Rayleigh-Taylor spikes and other jet-like structures.

Despite the absence of Kelvin-Helmholtz on jets, lift-driven motions (often labelled as 'Kelvin Helmholtz') do play an important role in certain respects. One sees in figure 1 the 'mushroom tips' on the spikes produced by Rayleigh Taylor. These develop because the faster flow around the end of the spike tip creates low pressure that pulls the material out laterally. In experiments or simulations with very simple sinusoidal initial conditions, the ends of the spike produce spiral rolls. This process has begun in some of the supernova-related experiments discussed above. Figure 15 shows an example. One can see this phenomenon very clearly in the simulation and less clearly in the data (because of limited resolution). 


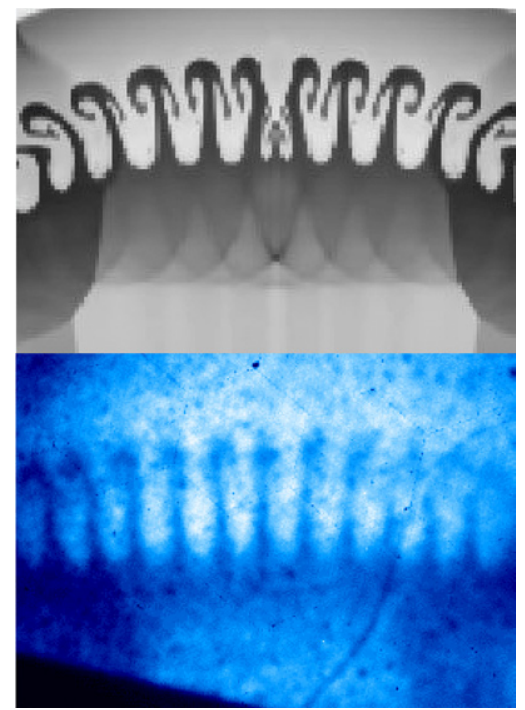

Figure 15. Simulation results by A Miles show Rayleigh-Taylor spikes on which the spike tips have been broadened to a 'mushroom' shape, also seen with less resolution in the experimental data of Robey et al [29,34], reprinted with permission American Institute of Physics.

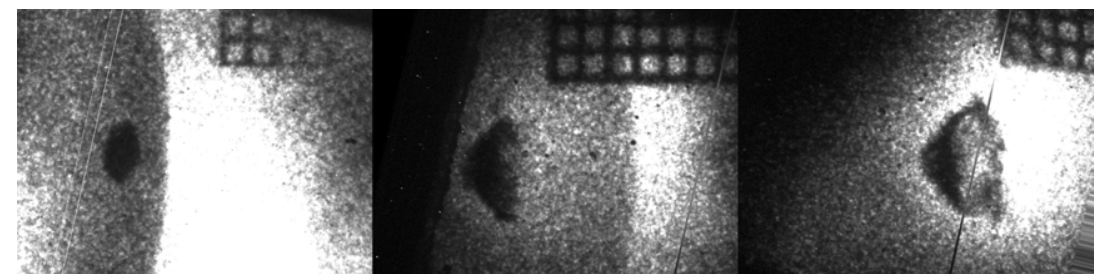

Figure 16. Destruction of a clump by a planar blast wave. From left to right, the blast wave has just passed the clump, the first vortex ring is being formed, and the vortex ring is separated from the clump (and is seen in another view to be developing modulations in azimuthal structure), from [47], with permission.

A second lift-driven effect plays a major role in the destruction of clumps by blast waves or more steady shocks, both of which occur in astrophysics and have been produced in the lab. As the flow is diverted around the clump, it pulls clump material sideways and then rolls it up and pulls it past the clump. This process is known as vortex shedding, as the structures that are pulled away are shaped like rings and contain vorticity, discussed below. Figures 16-18 show three examples. Figure 16 shows the destruction of a clump by a blast wave, in an experiment [47] designed to be relevant to supernova remnants like the Cygnus loop [48]. In the first frame the blast wave has just overtaken the clump squashing it in one direction while pulling it laterally as just described. In the second frame a vortex ring has been pulled away from the clump and in the third frame the ring has become modulated in the third (azimuthal) direction.

The next figures show results from an experiment [49-51] (figure 17) and a simulation $[52,53]$ (figure 18), both relating to the destruction of clumps by the more extended shock waves and plasma flows present in young supernova remnants, discussed above with reference to figure 7 . In the experiment of figure 17, an ejecta-driven shock wave and plasma flow was produced by the technique described in [54-56]. This flowing plasma overtook a clump of 


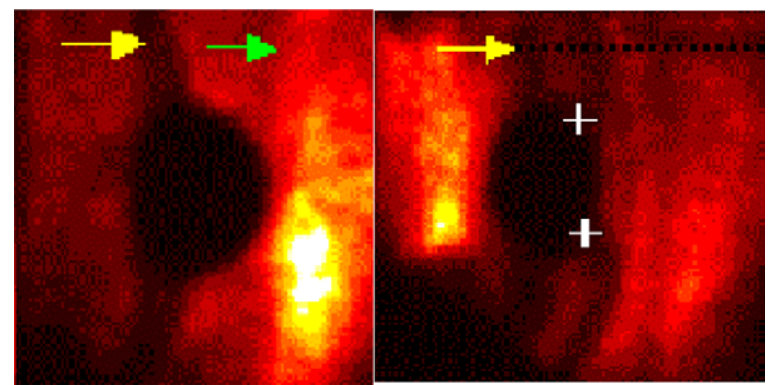

Figure 17. Interaction of a clump with extended flowing plasma, causing vortex shedding, adapted from [51].
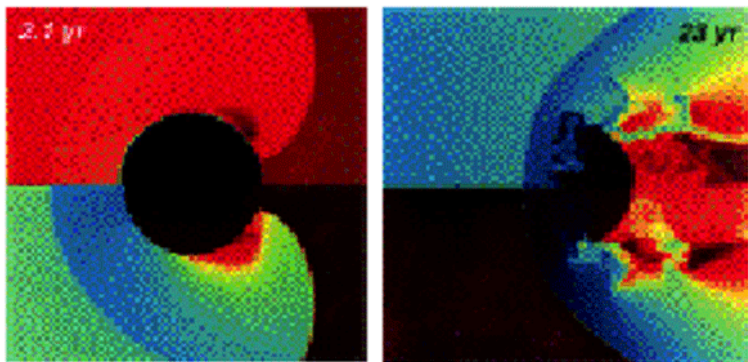

Borkowski et al., Ap. J. 477, 281 (1997)

Figure 18. Interaction of a model circular ring with the flowing plasma in a young supernova remnant, motivated by supernova SN 1987A, from [52], reproduced by permission of the AAS.

material, as can be seen in the first frame. The left arrow shows the trailing edge of the plasma flow, the right edge of the dark band is the contact surface and the right arrow shows the forward shock. In the second frame, the white crosses show where a vortex ring is being peeled off the clump. In the simulation of figure 18, a similar plasma flow is produced in a specific model of SN 1987A and overtakes the ring in that system, again specified by one specific choice of a model. One sees the forward shock and reverse shock in the first frame and in the second frame sees two of the vortex rings produced as the clump is destroyed. 2D models like that of figure 18 can produce the vortex shedding but will be unable to observe the subsequent development of structure in the third dimension or the onset of turbulence that may further mix the clump material into the medium.

\section{Vorticity effects}

Some plasma physicists work extensively with vorticity but many others never do. The vorticity is defined as the curl of the velocity, $\omega=\nabla \times \boldsymbol{u}$. It corresponds to swirling motions throughout volumes and also exists on surfaces when there is velocity shear across them. We have seen that velocity shear is required to produce the Kelvin-Helmholtz instability, which is thus integrally involved with surface vorticity. We did not discuss this, but the Rayleigh-Taylor instability acts to deposit vorticity on the surface between the two fluids. Even so, the major instabilities are instabilities - they have effects that provide feedback and naturally display exponential growth in time. Yet vorticity can have notable long-term effects even in the absence of instability, because once generated vorticity endures. 


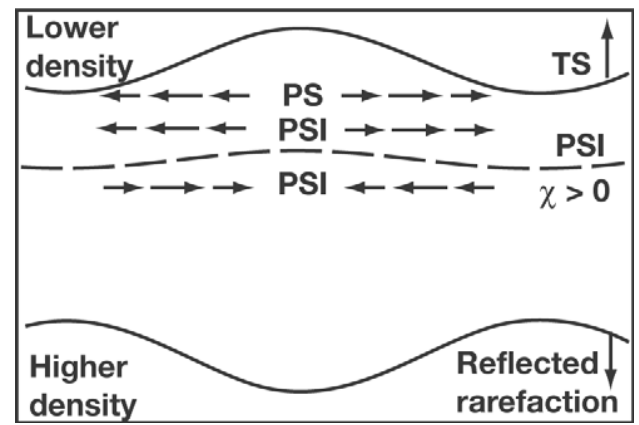

Figure 19. The Richtmyer-Meshkov process. A sketch of a system in which a planar shock encountered a rippled interface where there was a large decrease in density. The arrows show the lateral flow velocity, which acts to increase the size of the ripples, from [58].

By taking the curl of the Navier-Stokes equation (the Euler momentum equation with an added term for viscosity), one can develop the vorticity transport equation, which is

$$
\frac{\partial \omega}{\partial t}=\nabla \times(\boldsymbol{u} \times \omega)+v \nabla^{2} \omega
$$

in which the kinematic viscosity is $v$. This equation should be quite remarkable to a plasma physicist, as it is identical to the equation for the transport of the magnetic field. The analogy is not complete but is often useful. In particular, vorticity is frozen into a fluid just as the magnetic field is frozen into a plasma. And viscosity causes vorticity to diffuse just as resistivity causes magnetic diffusion. Vortical motion can be dissipated by viscous heating, but this typically occurs on very small spatial scales and first requires the generation of small-scale structures.

Vorticity is intimately involved in traditional hydrodynamic turbulence, which may develop in astrophysical systems and in some future high-energy-density experiments but is not evident in the existing data. So we will leave this topic for a future time. We will take up here a consequence of vorticity generation by shocks - the Richtmyer Meshkov 'instability' which is of substantial importance in laboratory environments and very likely also affects the evolution of shocked astrophysical systems.

Figure 19 illustrates the origin of the Richtmyer-Meshkov process when an initially uniform shock wave crosses a planar interface at which the density decreases substantially. Once the shock wave reaches the interface, a shock wave is driven into the low-density material while a rarefaction wave, such as the one discussed above with reference to the formation of blast waves, propagates backwards into the previously shocked material. If the density drop is large enough, the edge of the expansion of the higher-density material will be moving faster than the sound speed of the shocked higher-density material. This is the case shown in the figure. The ripple in the rarefaction wave is stable and propagates backwards through the material. The ripple in the shock wave is damped and dies out with time. The Richtmyer-Meshkov process is what happens at the interface.

The arrows in figure 19 show the lateral flow velocity just after the shock wave has crossed the interface. The effect of the ripple, which is the source of this lateral velocity, is to divert the shock wave and the reflected wave. One can see the lateral velocity will cause more material to flow into the protruding regions of dense material (the 'spikes'), causing them to grow and that similarly the 'bubbles' will also grow. If the density drop is smaller, the initial response of the flow is to oppose the ripples on the interface, causing them to invert and then grow with the opposite phase. In either event, the ripples on the interface grow in time after the passage 

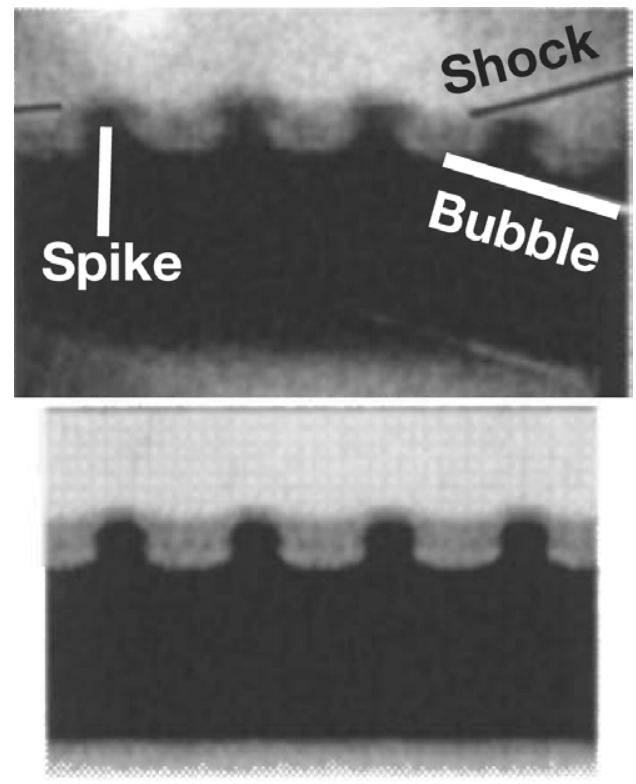

Figure 20. Experimental (above) and simulated (below) x-ray radiographs from an experiment in which a high-Mach-number shock encountered a rippled interface, adapted from [57].

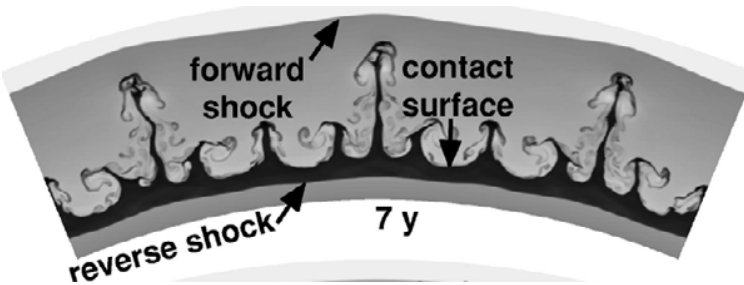

Figure 21. Greyscale image of density from a simulation in which a young supernova remnant encounters a rippled interface where the density drops, from [59], reproduced by permission of the AAS.

of a shock wave. They grow at first linearly in time and later more slowly. Similar dynamics occurs if the interface is planar and the shock is rippled or if both are rippled.

Richtmyer-Meshkov is important for inertial fusion, where structure in the irradiation of a capsule can produce rippled shocks and where shocks in the capsule may encounter structured interfaces (depending on how the capsule is made). This has motivated experiments. Figure 20 shows data and simulation from an experiment designed to measure the Richtmyer-Meshkov process [57]. In this experiment a steady planar shock wave overtook a rippled interface. There was no deceleration and thus there was no growth of the structure by the RayleighTaylor instability. Yet large structures were produced. In this case of a very strong shock, the growth of the spikes was so vigorous that they created a distortion of the shock.

The Richtmyer-Meshkov process also probably plays a role in astrophysics. Figure 21 shows a result from a simulation performed to demonstrate this. Both density clumps and density drops or increases can occur in circumstellar and interstellar environments, from a variety of causes. A model young supernova remnant (see figure 7) was simulated, producing an unstable contact surface like that of figure 1 . The shocked flow encountered a rippled 
density drop and then continued to propagate. The result was that the Richtmyer-Meshkov process influenced the structure of the spikes for a period of time. Specifically, it caused some of the spikes to penetrate much further towards the forward shock.

\section{Conclusion}

We have seen that a hydrodynamic description can sensibly be applied to a range of systems in astrophysics and high-energy-density experiments including inertial fusion systems. In consequence, buoyancy driven instabilities create a structure in all these systems whenever the entropy gradient is parallel to the pressure gradient. The most familiar and common example of this is the Rayleigh-Taylor instability, which occurs when the pressure gradient and density gradient are opposed. In both astrophysical and laboratory systems, this instability can develop in systems where there is ablative acceleration, in systems where there are ejectadriven shocks, and in systems in which blast waves encounter interfaces. The last two cases are substantially similar in their long-term development. Using laser facilities now under construction, it will become possible to address in the laboratory the complex processes that occur involving several interacting unstable interfaces in a diverging explosion like that in a supernova. Such experiments will go beyond conditions that can readily be simulated.

Lift-driven unstable process are also present, although the Kelvin-Helmholtz instability on jets or other interfaces is less common in astrophysics and in high-energy-density systems than it is in laboratory experiments with gasses and liquids. This presumably reflects the development of finite gradients in the flow velocity across the shear layer. Among the liftdriven effects that are prominent are the generation of mushroom-shaped tips on the spikes produced by Rayleigh-Taylor instabilities and vortex shedding during the destruction of clouds.

Vorticity effects are likewise important. The most common of these in inertial fusion and other laboratory experiments is the Richtmyer-Meshkov process, which leads to the growth of structures after a shock wave encounters a rippled interface. This process is also very likely to be important in astrophysical systems. The destruction of vortex rings by the development of unstable structure around the axis of the ring has been observed in the laboratory and is likely to be important in any system that generates vortex rings. The degree to which this may lead to the explosive growth of vorticity and the onset of turbulence remains a topic for future research.

\section{Acknowledgments}

The author acknowledges useful conversations on these topics with Sasha Velikovich, Harry Robey, Riccardo Betti, Roger Chevalier, Adam Frank, Jack Hughes, Dave Arnett, Dmitri Ryutov, Bruce Remington and interactions regarding experiments with Brent Blue, Freddy Hansen, John Foster and the Omega Jet Team. This research was sponsored by the National Nuclear Security Administration under the Stewardship Science Academic Alliances Program through DOE Research Grant DE-FG03-99DP00284 and other grants and contracts.

\section{References}

[1] Arnett W D 1996 Supernova and Nucleosynthesis (Princeton, NJ: Princeton University Press)

[2] Lindl J D 1998 Inertial Confinement Fusion (New York: Springer)

[3] Chevalier R A, Blondin J M and Emmering R T 1992 Astrophys. J. 392118

[4] Jun B I, Jones T W and Norman M L 1996 Astrophys. J. 468 L59

[5] Takabe H 1993 J. Plasma Fusion Res. 691285 (in Japanese) 
[6] Remington B A et al 1999 Science 2841488

[7] Drake R P 1999 J. Geophys. Res. 10414

[8] Remington B A et al 2000 Phys. Plasmas 71641

[9] Remington B A, Drake R P and Ryutov D D 2005 Rev. Mod. Phys. at press

[10] Landau L D and Lifshitz E M 1987 Fluid Mechanics (Oxford: Pergamon Press)

[11] Ryutov D D et al 1999 Astrophys. J. 518821

[12] Ryutov D D, Drake R P and Remington B A 2000 Astrophys. J. Suppl. 127465

[13] Ryutov D D et al 2001 Phys. Plasmas 81804

[14] Chavalier R A 1982 Astrophys. J. 258790

[15] Goncharov V N et al 2003 Phys. Plasmas 101906

[16] Remington B A et al 1993 Phys. Fluids B 42589

[17] Arnett D, Fryxell B and Muller E 1989 Astrophys. J. Lett. 34163

[18] Fryxell B, Muller E and Arnett D 1991 Astrophys. J. 367619

[19] Kifonidis K et al 2000 Astrophys. J. Lett. 531 L123

[20] Kifonidis K et al 2003 Astron. Astrophys. 408621

[21] Muller E, Fryxell B and Arnett D 1991 Astron. Astrophys. 251505

[22] Boehly T R et al 1995 Rev. Sci. Instrum. 66508

[23] Remington B A et al 1997 Phys. Plasmas 41994

[24] Kane J et al 1997 Astrophys. J. 478 L75

[25] Kane J et al 1999 Phys. Plasmas 62065

[26] Kane J et al 2000 Astrophys. J. Suppl. 127365

[27] Kane J O et al 2001 Phys. Rev. E 63 055401R

[28] Robey H F et al 2001 Phys. Plasmas 82446

[29] Robey H F et al 2003 Phys. Plasmas 10614

[30] Robey H F 2004 Phys. Plasmas 114123

[31] Robey H F et al 2004 Proc. Inertial Fusion Science and Applications 2003 (American Nuclear Society)

[32] Drake R P et al 2002 Astrophys. J. 564896

[33] Drake R P et al 2004 Phys. Plasmas 112829

[34] Miles A R et al 2004 Phys. Plasmas 113631

[35] Kuranz C C et al 2004 Astrophys. Space Sci. 2989

[36] Kuranz C C et al 2004 Astrophys. space Sci. 298267

[37] Calder A et al 2002 Astrophys. J. 143201

[38] Miles A R, Edwards M J and Robey H F 2004 Proc. Inertial Fusion Science and Applications 2003 (American Nuclear Society)

[39] Miles A 2004 PhD Thesis (University of Maryland, College Park)

[40] Miles A R et al 2004 Phys. Plasmas 113631

[41] Miles A R 2004 Phys. Plasmas 115140

[42] Miles A R, Edwards M J and Greenough J A 2004 Phys. Plasmas 115278

[43] Miles A R et al 2005 Phys. Plasmas 12

[44] Baclet P et al 1999 Int. Conf. on Fusion Science and Applications 99 ed C Labaune et al (Amsterdam: Elsevier) p 1083

[45] Benuzzi-Mounaix et al 2001 Astrophys. Space Sci. 277143

[46] Dimotakis P E et al 1983 Phys. Fluids 263185

[47] Robey H F 2002 Phys. Rev. Lett. 890850011

[48] Klein R I et al 2000 Astrophys. J. Suppl. Ser. 127379

[49] Kang Y G et al 2000 Proc. SPIE 3886489

[50] Kang Y G et al 2001 Plasma Phys. Rep. 27843

[51] Kang Y G et al 2001 Phys. Rev. E 64047402

[52] Borkowski K J, Blondin J M and Mccray R 1997 Astrophys. J. 477281

[53] Borkowski K J, Blondin J M and Mccray R 1997 Astrophys. J. 476 L31

[54] Drake R P et al 1998 Phys. Rev. Lett. 812068

[55] Drake R P et al 1998 Astrophys. J. Lett. 500 L157

[56] Drake R P et al 2000 Astrophys. J. Suppl. 127305

[57] Glendinning S G et al 2003 Phys. Plasmas 101931

[58] Drake R P 2006 High Energy Density Physics: Foundation of Inertial Fusion and Experimental Astrophysics (Berlin: Springer)

[59] Kane J, Drake R P and Remington B A 1999 Astrophys. J. 511335 